Research Paper

\title{
Hypoxia Enhances Stemness of Cancer Stem Cells in Glioblastoma: An In Vitro Study
}

\author{
Pengcheng $\mathrm{Li}^{1}$, Chun Zhou ${ }^{1}$, Lunshan $\mathrm{Xu}^{1 凶}$, Hualiang Xiao ${ }^{2 \bowtie}$ \\ 1. Department of Neurosurgery, Research Institute of Field Surgery, Daping Hospital, Third Military Medical University, Chongqing, \\ 400042, China. \\ 2. Department of Pathology, Research Institute of Field Surgery, Daping Hospital, Third Military Medical University.
}

\begin{abstract}
$\triangle$ Corresponding author: Lunshan Xu and Hualiang Xiao; xulunshan@dphospital.tmmu.edu.cn; xiaoyyuk@163.com.
(c) Ivyspring International Publisher. This is an open-access article distributed under the terms of the Creative Commons License (http://creativecommons.org/ licenses/by-nc-nd/3.0/). Reproduction is permitted for personal, noncommercial use, provided that the article is in whole, unmodified, and properly cited.
\end{abstract}

Received: 2012.10.18; Accepted: 2013.02.11; Published: 2013.02.21

\begin{abstract}
Objective: To investigate the relationship between hypoxia and in vitro "stemness" of cancer stem cells (CSCs). Methods: U87 cells, U25 I cells and primary glioma cells $(n=3)$ experienced hypoxia. Transmission electron microscopy was done to detect the ultrastructure of these cancer cells; MTT assay to detect the cell growth; flow cytometry to detect cell cycle and CDI33 expression; Transwell chamber assay was carried out to detect the cell migration; colony-forming assay to detect the colony-forming efficiency; real-time quantitative PCR and Western blot were carried out to detect the mRNA and protein expression of markers of stem cells and their differentiation, respectively. Results: Hypoxia maintained the undifferentiated state of primary glioma cells, slowed down the growth of glioma cells which were in a relatively quiescent stage, increased the colony forming efficiency and migration of glioma cells, and elevated the expression of markers of stem cells, but the expression of markers for stem cell differentiation was reduced after hypoxia treatment. Conclusion: Hypoxia may induce the "dedifferentiation" of differentiated glioma cells which then acquire the stemness.
\end{abstract}

Key words: Cancer stem cell; Stemness; Hypoxia; Gioblastoma; Invasiveness.

\section{Introduction}

Glioma is a major tumor in the central nervous system and accounts for $35-50 \%$ of intracranial tumors in adults. In addition, malignant glioma accounts for about $60 \%$ of glioma [1]. Currently, surgical intervention is still the major strategy for the treatment of glioma, with adjuvant radiotherapy, chemotherapy and biotherapy. However, the therapeutic efficacy is still unsatisfactory. In Department of Neurosurgery, standard therapy in combination with chemotherapy and/or radiotherapy may achieve a mean survival time of 14.6 months [2].

In recent years, increasing evidence confirms that cancer is a disease with involvement of stem cells. It is the large amount of cancer stem cells (CSCs) that promote growth of cancer and facilitate invasion, re- currence and metastasis of cancers [3]. In the light of the important role of CSCs in occurrence and development of cancers, CSCs may have promise to become an idea target in the treatment of cancers. However, more studies are required to elucidate the growth and differentiation of cancer cells, especially the interaction between cancer cells and microenvironment of cancers.

Hypoxia is an important feature of malignancies including glioma. Previous work focused on the effect of hypoxia on angiogenesis and energy metabolism in cancers, and less attention has been paid to the influence of hypoxia on growth and differentiation of cancer cells, especially the cancer stem cells [4]. On the basis of previous studies, we hypothesized that hy- 
poxia played important roles in regulation of growth and differentiation of CSCs. The present study aimed to investigate the interaction between CSCs and microenvironment in cancers, which may help to understand the mechanism underlying the regulation of differentiation of CSCs. Our findings may be beneficial for identifying factors targeting the hypoxia and CSCs in cancers, which may provide experimental evidence for the targeted therapy of gioblastoma.

\section{Materials and methods}

\section{Isolation, identification and culture of CSCs}

The isolation of CSCs and CD133-positive cancer cells was done with magnetic activated cell positive sorting (MACS) according to previously described [5]. In brief, fresh cancer tissues were obtained and washed. Necrotic tissues and blood were removed. Then, cancer tissues were cut into blocks $\left(1 \times 1 \times 1 \mathrm{~mm}^{3}\right)$ followed by digestion in trypsin and centrifugation. The corresponding cells were harvested and grown in serum free medium containing bFGF and EGF. Then, primary neurospheres were collected and subjected to flow cytometry to collect CD133 positive and CD133 negative cancer cells followed by further culture.

\section{Detection of cell cycle}

U87 cells were incubated in normoxia or $1 \% \mathrm{O}_{2}$. At different time points, cells were collected and subjected to flow cytometry to detect the cell cycle.

\section{Magnetic activated cell positive sorting}

MACS is a newly developed method for sorting cells. With this method, marker of target cells binds to the microbeads and these cells were sorted under a magnetic condition. In our previous studies, CD133 positive CSCs and gioblastoma derived endothelial cells were successfully sorted with this method $[5,6]$. The CD133 microbeads and goat anti-mouse IgG microbeads were purchased from Miltenyi, Germany.

\section{MTT assay}

MTT assay was employed to detect the growth of glioma cells and the growth curve was delineated. Logarithmic phase cells were collected, and the concentration of the cell suspension was adjusted to 5000 cells per well (The edge wells of the plate are filled with aseptic PBS buffer). The cells were incubated at $37^{\circ} \mathrm{C}, 5 \% \mathrm{CO} 2$ until cells cover the bottom of the well (a flat-bottom 96-well plate), and then the cells were cultured under normoxic or hypoxic conditions. $20 \mathrm{ul}$ of the MTT solution was added to each well $(5 \mathrm{mg} / \mathrm{ml}$, $0.5 \% \mathrm{MTT}$ ) and the cells were continued to culture for 4h. After the incubation, the supernatant was discarded and 150 ul Dimethyl sulfoxide was added to each well, and the culture plate was shaked at low speed for 10min until crystal dissolved completely. The ELISA reader was used to measure the absorbance at $570 \mathrm{~nm}$.

\section{Colony forming assay}

U251 cells in logarithmic growth phase were digested in $0.5 \%$ trypsin / $0.04 \%$ EDTA and single cell suspension was prepared. Then, these cells were added to 24-well plates (50 cells/well) followed by incubation at $37^{\circ} \mathrm{C}$ in an environment with saturated humidity and $5 \% \mathrm{CO}_{2}$ for $24 \mathrm{~h}$. Non-adherent cells were removed. After culture for 2-3 weeks, large colonies were present and then digested in $0.5 \%$ trypsin / $0.04 \%$ EDTA for preparation of single cell suspension. These cells were seeded into 96-well plates followed by incubation at $37^{\circ} \mathrm{C}$ in an environment with saturated humidity and $5 \% \mathrm{CO}_{2}$. The colony forming efficiency and the morphology of colonies were observed. The size of colonies was measured and cells in each colony were counted. At this time, cells were divided into early passaging group and late passaging group. In the early passaging group, cells with target number were digested in $0.5 \%$ trypsin / $0.04 \%$ EDTA for preparation of single cell suspension. These cells were then seeded into 96-well plates. In the late passaging group, cells were digested in $0.5 \%$ trypsin / $0.04 \%$ EDTA for preparation of single cell suspension. These cells were then seeded into 96-well plates.

\section{Transwell migration assay}

$40 \mu \mathrm{l}$ of artificial basement membrane (matrigel + serum free medium) was added to the upper well followed by incubation at $37^{\circ} \mathrm{C}$ overnight. Then, the cells $\left(1 \times 10^{6} / \mathrm{ml}\right)$ were added to the upper well $(100$ $\mu \mathrm{l})$, and conditioned medium $(600 \mu \mathrm{l})$ was added to the lower well followed by incubation for $24 \mathrm{~h}$. The transwell was taken out, and the medium in the upper well was removed. Then, the upper well was washed in PBS once. The matrigel and residual cells in the upper well were swabbed. After washing in PBS twice, the upper well was fixed in $4 \%$ paraformaldehyde for $30 \mathrm{~min}$. After washing in PBS twice, the upper well was stained with $0.1 \%$ crystal violet for 20 min. Following washing in ddH2O more than three times, the upper well was dried, and observed under a light microscope. Three fields were randomly selected and the cells were counted.

\section{Immunofluorescence staining}

U87 neurospheres in serum free medium were maintained on the polylysine pre-coated coverslips for $2 \mathrm{~h}$ followed by staining with GFAP or OCT4 and the subsequent observation under a laser confocal 
microscope. These cells were positive for GFAP, but negative for OCT4. After incubation of U87 cells in a hypoxic environment for 48, staining of GFAP, OCT4, nestin and SOX2 was done followed by observation under a laser confocal microscope. Cells positive for GFAP reduced, but they were positive for OCT4, nestin and SOX2.

\section{Western blot assay and Real-time quantitative PCR}

At different time points, the total protein was extracted from U87 cells and then subjected to SDS-PAGE and transferred onto nitrocellulose filter (Bio-Rad). The membrane was blocked in 5\% non-fat milk and then incubated with mouse anti-human CA IX, Oct-4, Sox- 2 or GFAP polyclonal antibody (Abcam, UK) or mouse anti-human $\beta$-actin monoclonal antibody (Sigma). Enhanced chemiluminescence (Pierce, Rockford, IL) was employed for visualization. Total RNA was extracted with Tripure method and real-time quantitative PCR was employed to detect the mRNA expression of OCT-4, SOX-2 and GFAP.

\section{Statistical analysis}

Statistical analysis was done with STATA 12.0. Data were expressed as mean \pm standard deviation. Comparisons between two groups were done with $t$ test. A value of $\mathrm{P}<0.05$ was considered statistically significant.

\section{Results}

\section{Effect of hypoxia on morphology of glioma cells}

Herein, we described the morphology of primary glioma cells (x01GBM) as a representative. As shown in Figure 1A, the $x 01 G B M$ cells were long-spindle, had processes and evenly distributed. After incubation in 1\% O2 for $24 \mathrm{~h}$, the morphology remained unchanged. After incubation in 1\% $\mathrm{O} 2$ for $48 \mathrm{~h}$, the cell processes became unobvious and cells were polygonal. After incubation in $1 \% \mathrm{O} 2$ for $72 \mathrm{~h}$, the processes were absent, and oval cells aggregated in masses (Figure 1B). However, these cells had favorable refractivity, the medium was clear and necrosis was not evident. Transmission electron microscopy showed the $x 01 G B M$ in routine medium were rich in organelles, collagen fibers were present, the nucleus was large-spindle and irregular, and the nucleolus was difficult to identify (Figure 1C-D). After incubation in $1 \% \mathrm{O} 2$ for $24 \mathrm{~h}$, the collagen fibers reduced, and some mitochondria were present, the nucleus was still irregular, and asymmetrical nucleolus was observed (Figure 1E-F). After incubation in 1\% O2 for $72 \mathrm{~h}$, the organelles reduced, the collagen fibers were absent, nucleolus was present in most cells and double nucleoli were present in a fraction of cells (Figure 1G-H). The changes in the morphology of U87 cells, U251 cells and $\mathrm{x} 02 \mathrm{GBM}$ were similar to those above.

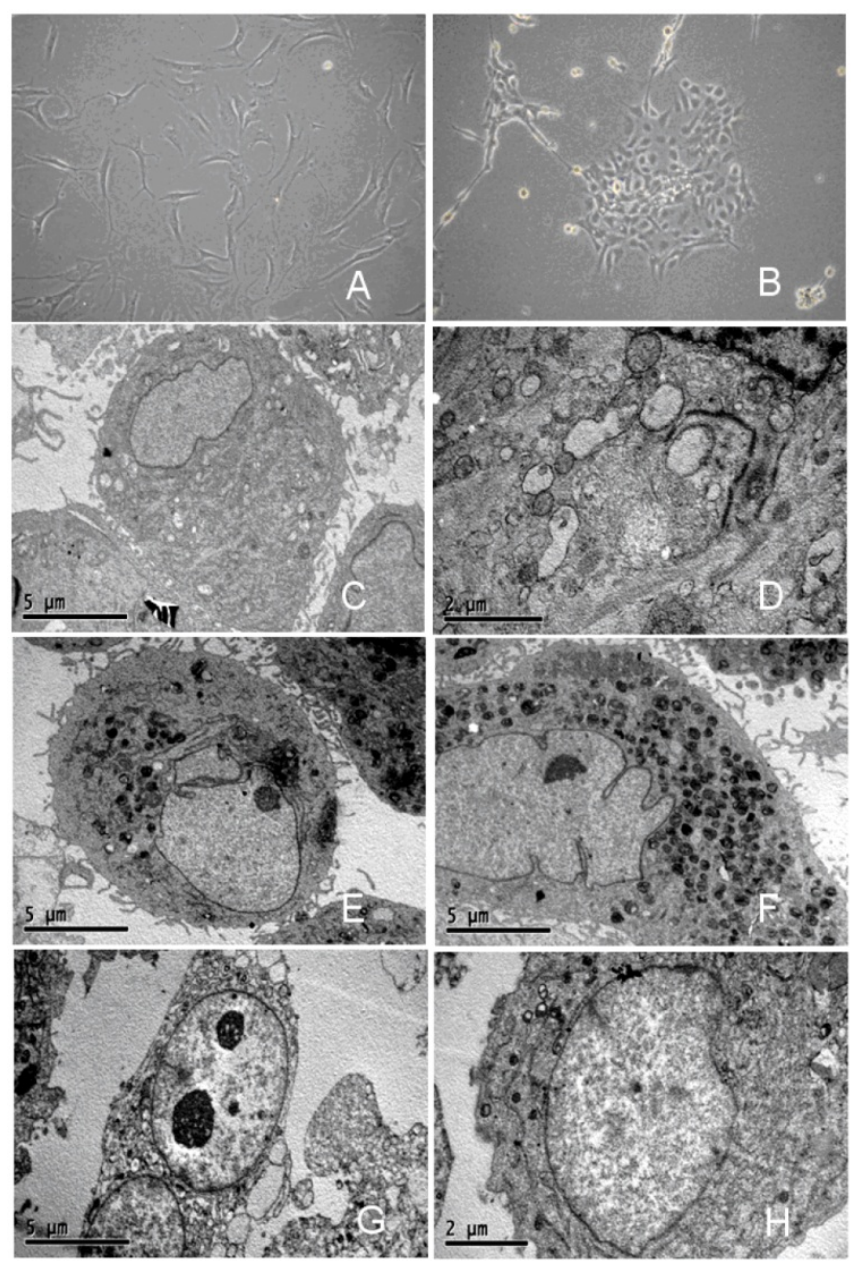

Figure I. Influence of hypoxia on morphology of glioma cells. A: x0IGBM in routine culture; B: hypoxic treatment for $72 \mathrm{~h}$; C-D: Ultrastructure of $\times 01 \mathrm{GBM}$ in routine culture; $\mathrm{E}-\mathrm{F}$ : Ultrastructure after hypoxic treatment (I\% O2) for $24 \mathrm{~h}$; G-H: Ultrastructure after hypoxic treatment ( $1 \%$ O2) for $72 \mathrm{~h}$.

\section{Effect of hypoxia on growth, cell cycle}

MTT assay was employed to detect the growth of glioma cells and the growth curve was delineated. Figure 2A displayed the growth curve of U87 cells and $\mathrm{x} 02 \mathrm{GBM}$. Results revealed that hypoxia could slightly inhibit the growth of cancer cells without significant difference. Figure 2B displayed the cell cycle. Results indicated that treatment with $1 \%$ O2 for 2, 12 and $24 \mathrm{~h}$ had no influence on the cell cycle. However, after treatment with $1 \% \mathrm{O} 2$ for 48 , the cells in G0/G1 phase significantly increased when compared with 
the control group $(\mathrm{P}<0.05)$, and those in the $\mathrm{G} 2 / \mathrm{M}$ phase markedly reduced $(\mathrm{P}<0.01)$. The changes in cell cycle were similar to those above.

\section{Effect of hypoxia on colony forming efficiency of glioma cells}

Colony forming assay showed hypoxia had significant influence on the colony forming efficiency of glioma cells (Figure 3). For U251 cells, the colony forming efficiency was significantly higher than that in the control group after hypoxic treatment $(\mathrm{P}<0.05)$. Especially, the proportion of compact colonies increased markedly. Under the hypoxic condition, the proportion of compact colonies in U251 cells was significantly higher than that in the control group $(\mathrm{P}<0.01)$.

\section{Effect of hypoxia on migration of glioma cells}

Transwell assay was employed to detect the cell migration. For U87 cells, the number of cells migrat- ing across the upper well was comparable to the control group after hypoxic treatment for $2 \mathrm{~h}$. Following hypoxic treatment for $12 \mathrm{~h}$ and $24 \mathrm{~h}$, the number of cells migrating across the upper well increased significantly when compared with the control group $(\mathrm{P}<0.05)$ (Figure 4).

\section{Effect of hypoxia on markers of CSCs and their differentiation}

\section{Effect of hypoxia on CDI33 expression}

After treatment with 1\% O2 for 48 h, CD133 expression was detected. When compared with the control group, hypoxia significantly increased the CD133 expression and the number of CD133 positive cells elevated markedly (Figure 5C). For x01GBM, the proportion of CD133 positive cells was $4.38 \pm 0.95(n=3)$ in the control group and $15.90 \pm 1.38(n=3)$ after hypoxic treatment for $48 \mathrm{~h}$ showing significant difference between two groups $(\mathrm{P}<0.01$; Figure $5 \mathrm{~A}-\mathrm{C})$.
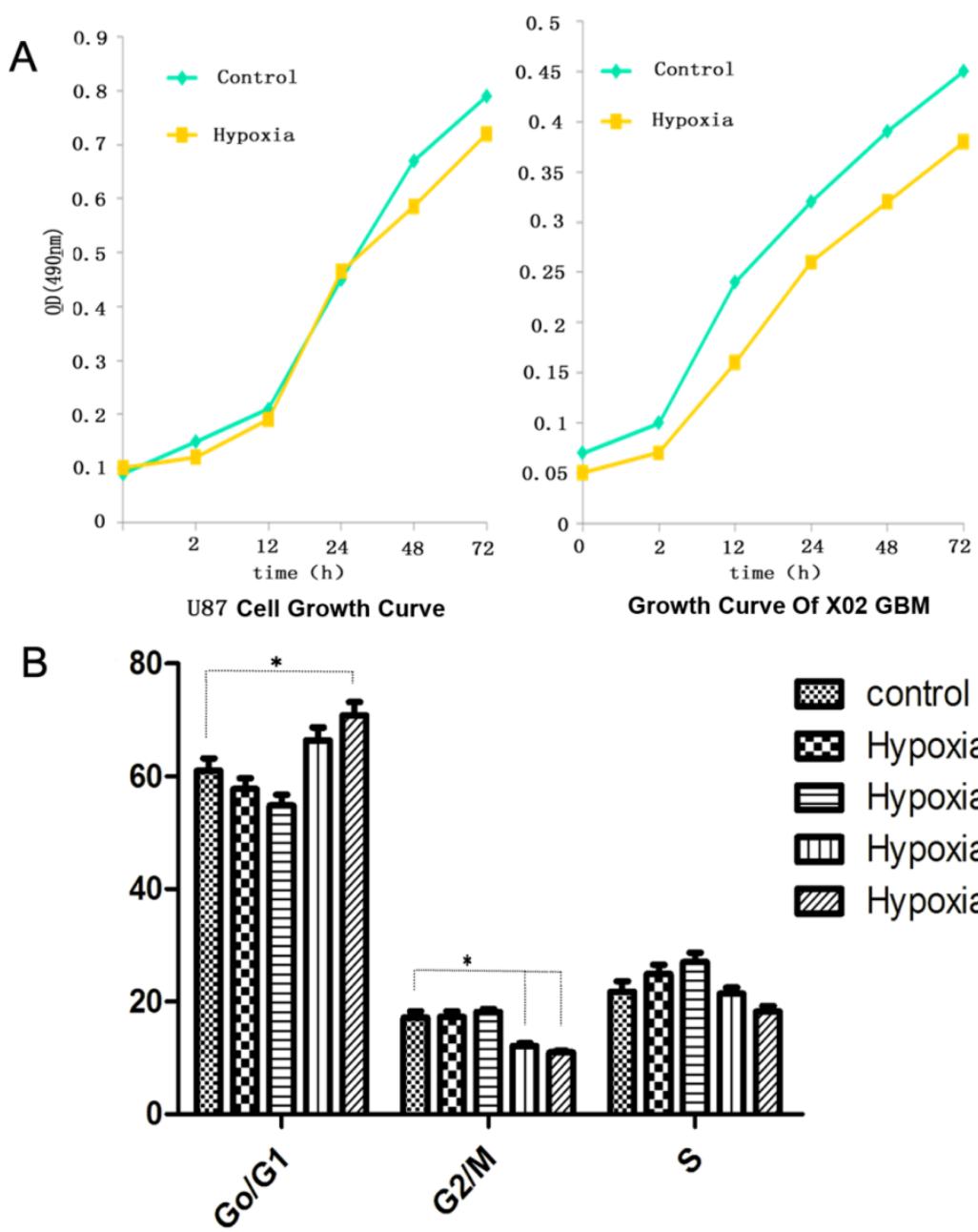

m control

x. Hypoxia2

目 Hypoxia12

س

Hypoxia48

Figure 2. Influence of hypoxia on growth and cell cycle of glioma cells. A: Growth curve of U87 cells and x0 IGBM; B: U87 cells in G0/G I phase, G2/M phase and S phase after hypoxic treatment $(1 \%$ O2) for $2,12,24$ and $48 \mathrm{~h}(* \mathrm{P}<0.05 ; * * \mathrm{P}<0.01 ; \mathrm{n}=3)$ 

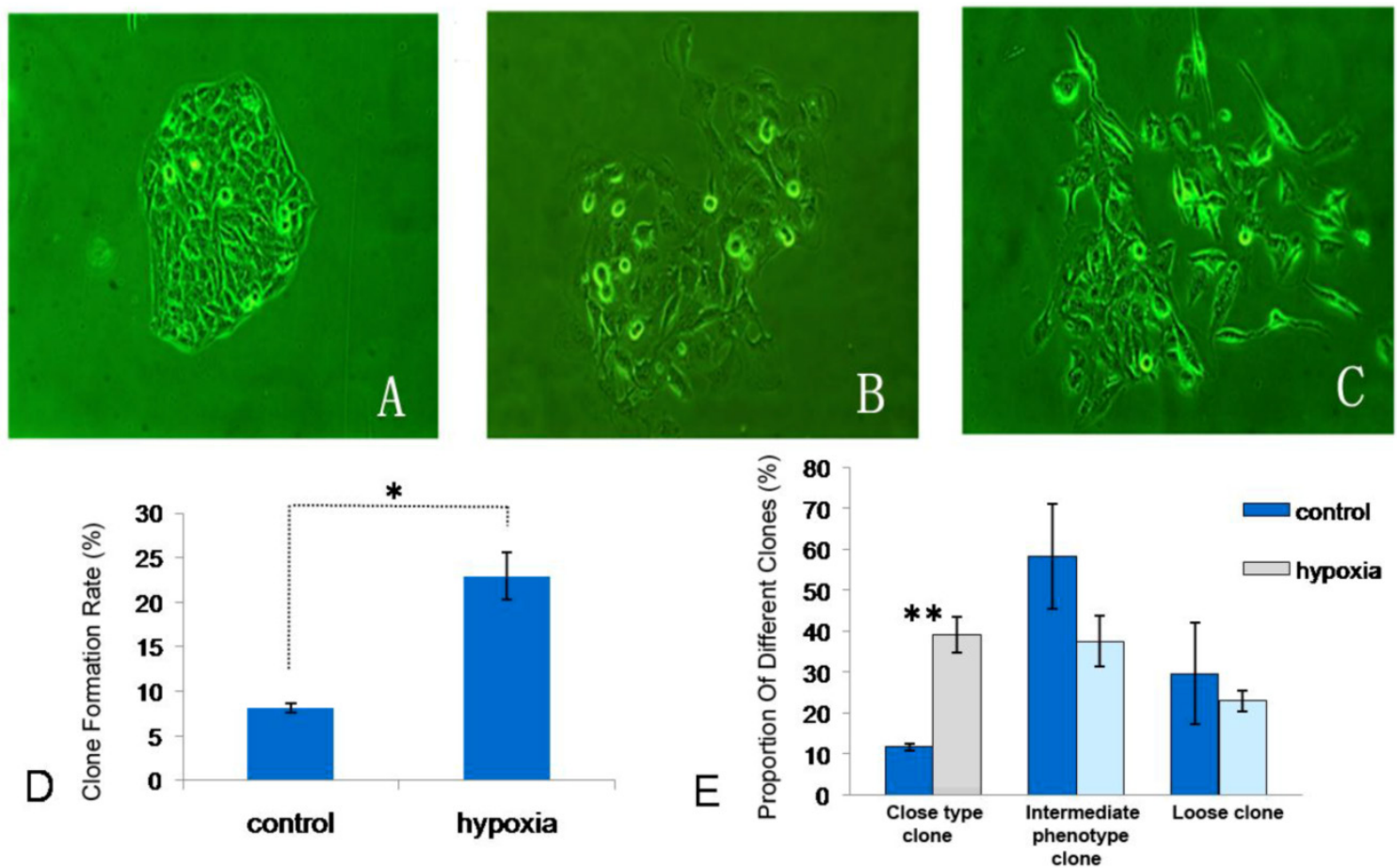

Figure 3. Influence of hypoxia on colony forming efficiency of U25I cells. A: compact colonies; B: intermediate colonies; C: loose colonies; D: influence of hypoxia on colony forming efficiency of U25I cells; E: Comparisons of colonies of different types of U25 I cells under normoxia and hypoxic conditions $(* \mathrm{P}<0.05 ; * * \mathrm{P}<0.0 \mathrm{I})$
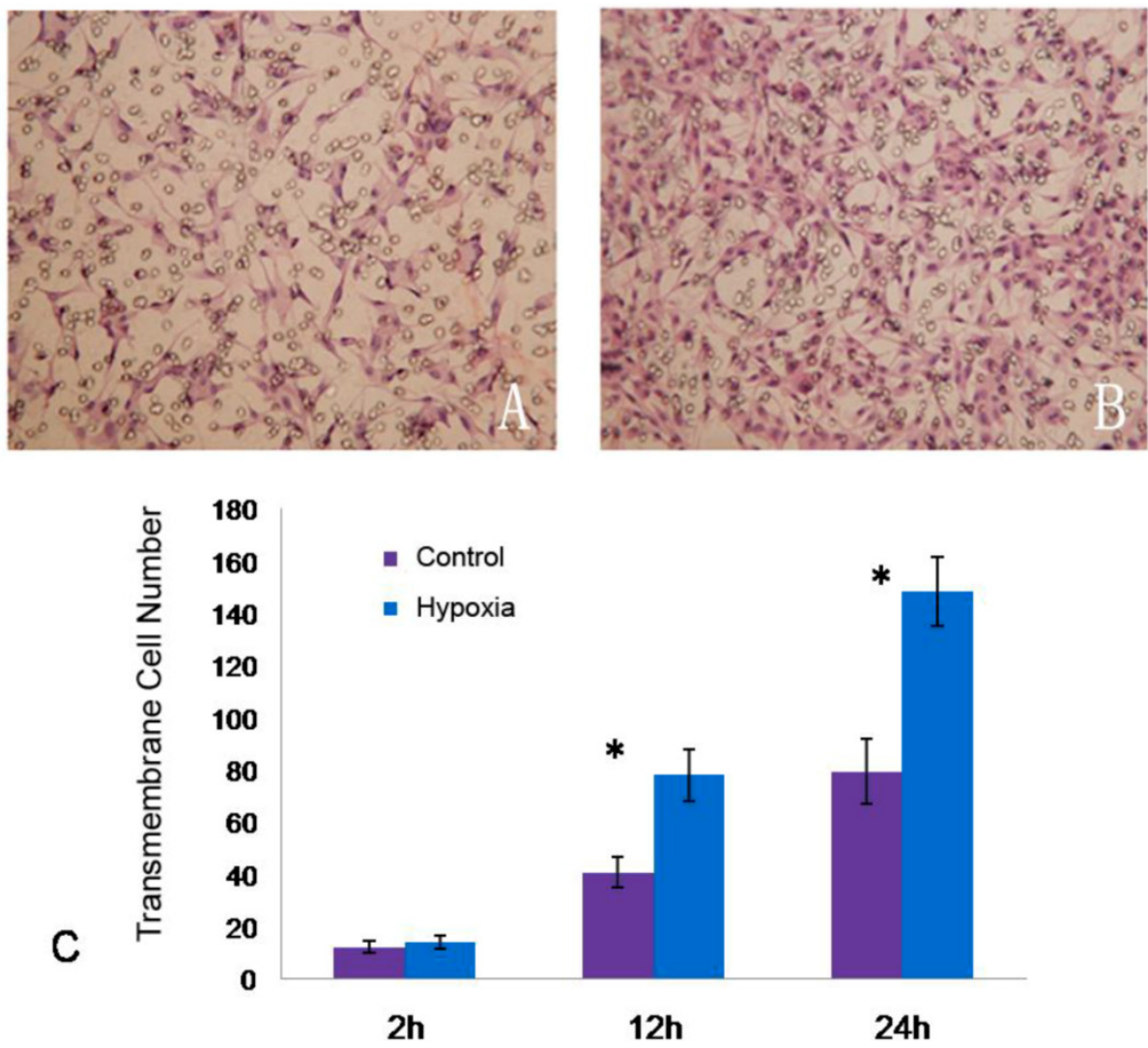

Figure 4. Influence of hypoxia on migration of U87 cells. A: U87 cells migrating the upper well after normoxia treatment for 24 h; B: U87 cells migrating the upper well after hypoxic treatment $\left(1 \% \mathrm{O}_{2}\right)$ for $24 \mathrm{~h}$; C: Comparison of cells migrating the upper well under normoxia and hypoxic conditions for 2,12 and $24 \mathrm{~h}(* \mathrm{P}<0.05)$. 

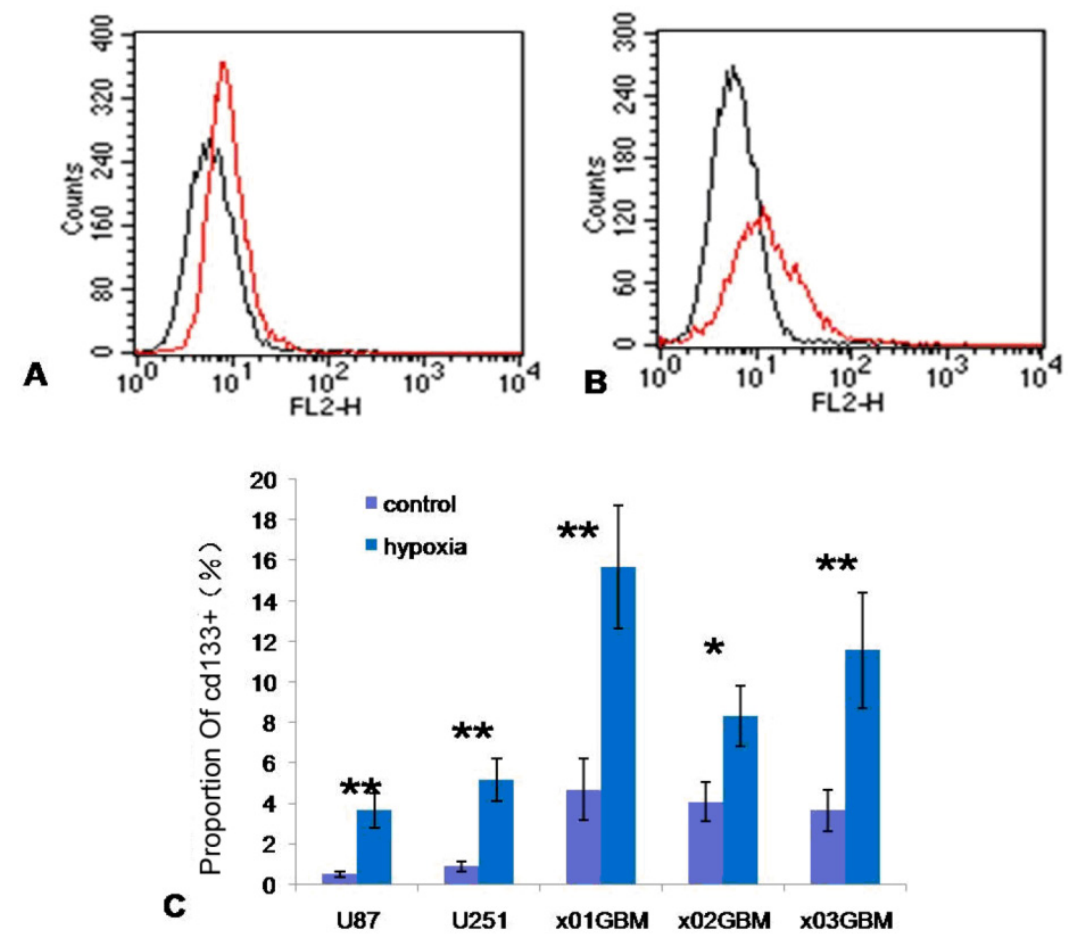

Figure 5. Influence of hypoxia on CDI33 expression in glioma cells. A: CDI33 expression in x0IGBM under normoxia condition; B: CDI 33 expression in $x 0$ IGBM under hypoxic condition for 48 h; C: the proportion of CDI 33 positive cells following hypoxic treatment $\left(\mathrm{I} \% \mathrm{O}_{2}\right)$ increased significantly when compared with the control group $(* \mathrm{P}<0.05$, **P<0.0I).

Effect of hypoxia on mRNA expression of markers of CSCs and their differentiation

Real-time quantitative PCR was employed to detect the markers of CSCs (OCT-4, SOX-2) and markers of differentiation of CSCs (GFAP). The amplification curve of OCT-4 (Figure 6A) was smooth, had evident exponential growth phase, linear growth phase and plateau phase, suggesting specific amplification. The mRNA expression of OCT-4, SOX-2 and GFAP in U87 cells at different time points is shown in Figure 6B-D. In the control group, the U87 cells had no mRNA expression of OCT-4 and SOX-2. After hypoxic treatment $(1 \% \mathrm{O} 2)$ for 2,12 and $24 \mathrm{~h}$, the mRNA expression of OCT- 4 and SOX-2 increased; after hypoxic treatment for $48 \mathrm{~h}$ and $72 \mathrm{~h}$, the mRNA expression of OCT-4 and SOX-2 elevated markedly when compared with other groups $(\mathrm{P}<0.01)$. However, the changes in mRNA expression of GFAP were different from those above. After hypoxic treatment for $2 \mathrm{~h}$ and $12 \mathrm{~h}$, the mRNA expression of GFAP was dramatically reduced and it remained at a low level after 48-h hypoxic treatment. After hypoxic treatment for $48 \mathrm{~h}$ and $72 \mathrm{~h}$, the mRNA expression of GFAP in U87 cells was significantly lower than that in the control group and in cells after hypoxic treatment for $2 \mathrm{~h}(\mathrm{P}<0.01)$. In the presence of pretreatment with DAPT $(0.5 \mu \mathrm{g} / \mathrm{ml}$; a specific inhibitor of Notch signaling pathway), the
mRNA expression of OCT-4 and SOX-2 in U87 cells after hypoxic treatment for $72 \mathrm{~h}$ was significantly lower than that in U87 cells with hypoxic treatment alone $(\mathrm{P}<0.01)$, but the mRNA expression of GFAP presented with inverse changes $(\mathrm{P}<0.01)$.

Detection of markers of CSCs and their differentiation by immunofluorescence staining

Double immunofluorescence staining showed the U87 cells in the control group had GFAP expression but had no expression of OCT-4 (Figure 7-A-D) and SOX-2. After hypoxic treatment for $48 \mathrm{~h}$, the GFAP expression reduced, the Nestin expression increased, and most cells had protein expression of OCT-4 and SOX-2. Similar changes were observed in other cells.

\section{Detection of markers of CSCs and their differentia- tion by Western blot assay}

As shown in Figure 8, Western blot assay showed the CA IX protein expression up-regulated in U87 cells after hypoxic treatment, and the protein expression of OCT-4 and SOX-2, two markers of CSCs, had increasing tendency. However, the GFAP protein expression reduced significantly after hypoxic treatment. Similar changes were observed in other cells. 
A
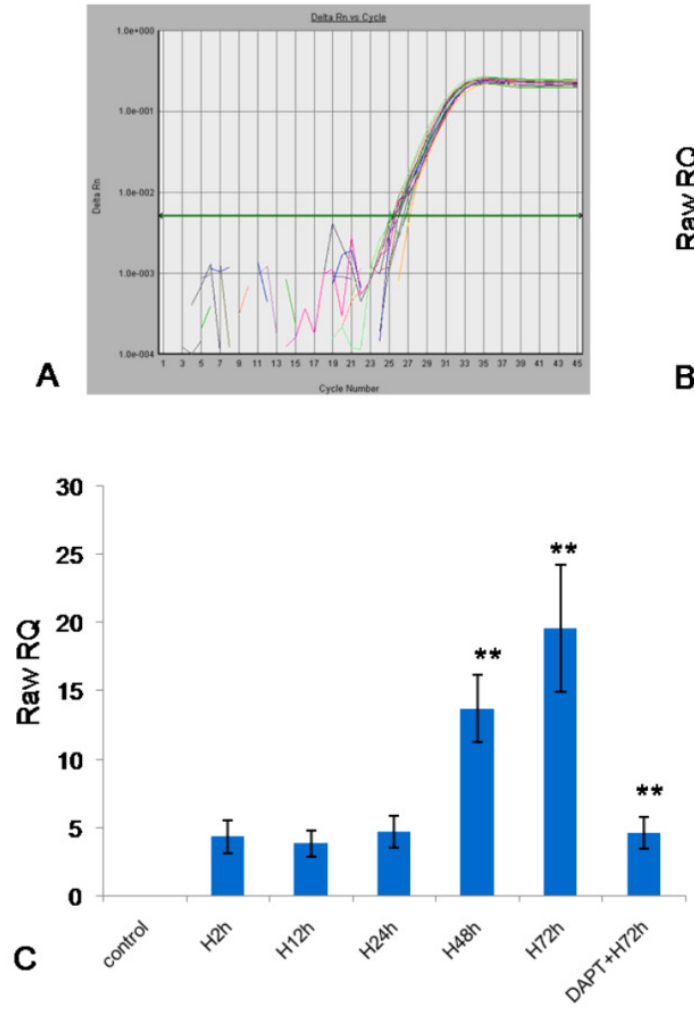
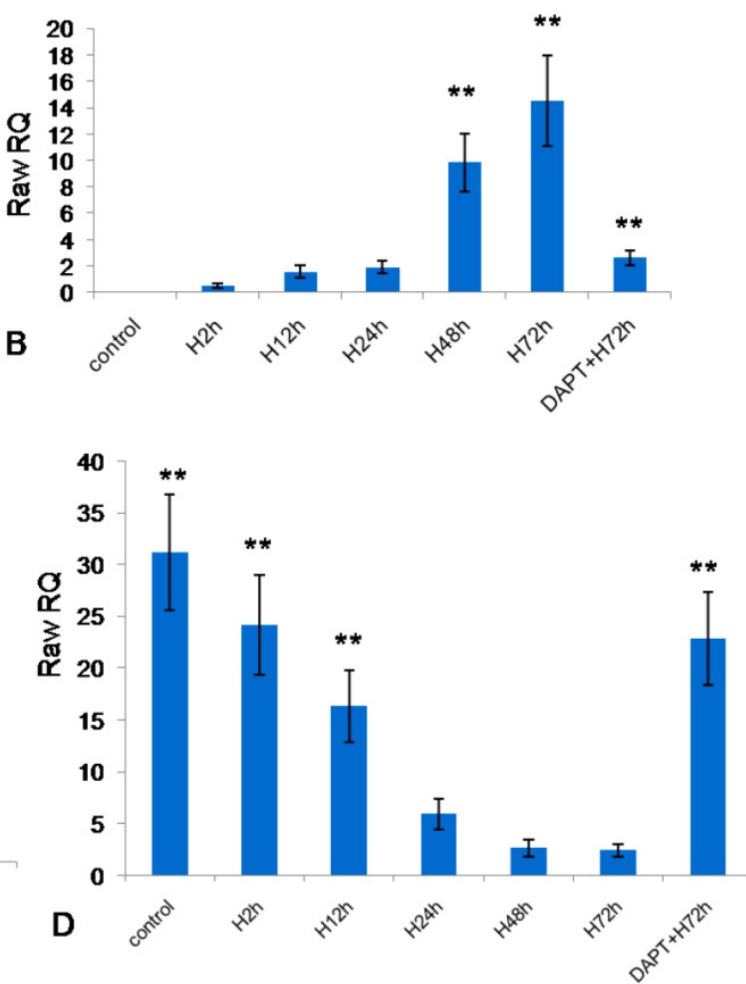

Figure 6. Influence of hypoxia on mRNA expression of OCT-4, SOX-2 and GFAP. A: amplification curve of OCT-4; B: mRNA expression of OCT-4 in U87 cells; C: mRNA expression of SOX-2 in U87 cells; D: mRNA expression of GFAP in U87 cells $(* P<0.05$, $* * P<0.01)$.

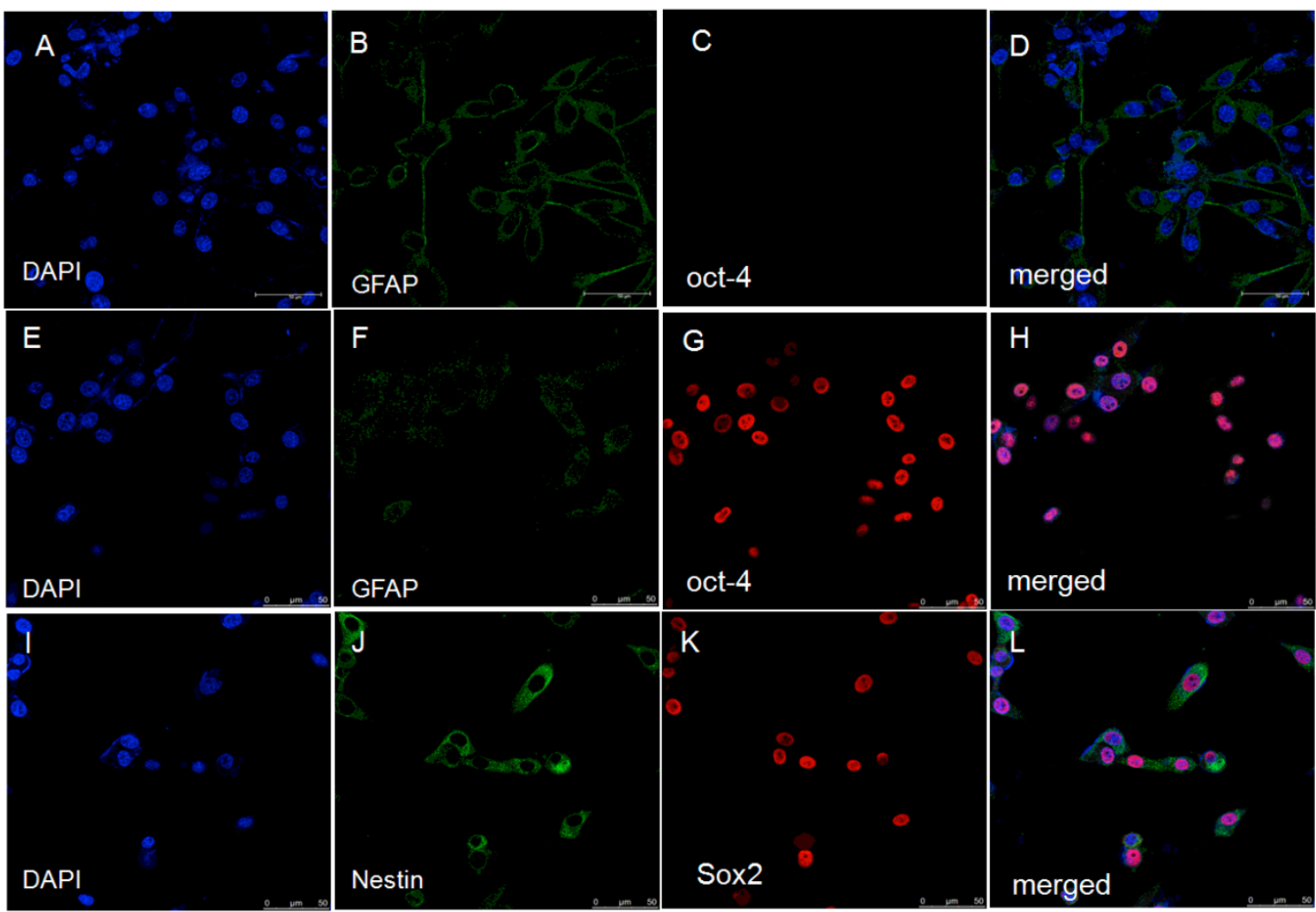

Figure 7. Double immunofluorescence staining showed up-regulation of markers of stem cells (OCT-4, SOX-2) and down-regulation of GFAP in U87 cells. A-D: U87 cells in control group; E-L: U87 cells after hypoxic treatment for 48 h. 


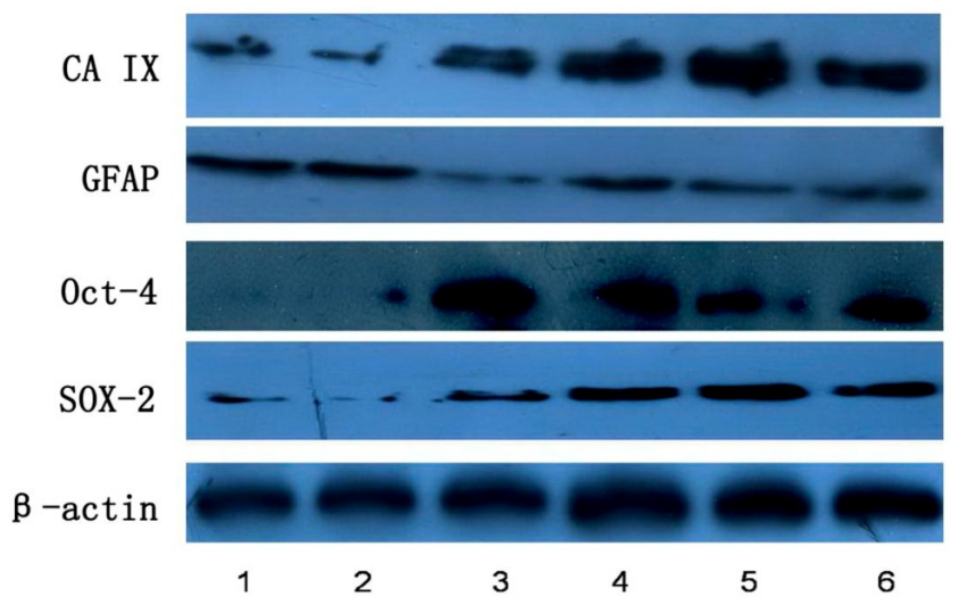

Figure 8. Influence of hypoxia on protein expression of CA IX, GFAP, OCT-4, and SOX-2. I: control; 2: Hypoxia for 2 h; 3: Hypoxia for 12 h; 4: Hypoxia for 24 h; 5: Hypoxia for 48 h; 6: Hypoxia for 72 h.

\section{Discussion}

Hypoxia is a common physiological and pathophysiological phenomenon. A series of complicated mechanisms are developed in human body to accommodate hypoxia. For example, glycolysis increases to compensate the energy deficiency due to compromised oxidative phosphorylation under the hypoxic condition [7]; angiogenesis increases under the hypoxic condition to increase the blood vessel density and subsequently elevate the oxygen supply; systemic hypoxia may increase the expression of erythropoietin as a physiological response to accommodate the hypoxic environment. In addition, hypoxia is also a common pathology. For example, hypoxia is a common phenomenon in cancers [8].

Previous studies has shown that hypoxia may induce the expression of hypoxia-inducible factors (HIF-1a), which may regulate the expression of different target genes affecting the growth and proliferation of cancer cells, and angiogenesis in cancers [9-11]. In recent years, results also reveal that hypoxia is related to the maintenance of undifferentiated state of stem cells and the proliferation and differentiation of stem cells in the neural crest and central nervous system [12, 13]. The hypoxia related regulation of stem cells has involvement of some stem cell related signaling molecules. To date, the signaling molecules involving in the normal self-renew and differentiation of stem cells include Wnt, BMP, Notch and Sonic hedgehog (Shh). Recent studies indicate that hypoxia may activate HIF-1a to up-regulate the down-stream molecules in Notch signaling pathway, which is crucial for the maintenance of undifferentiated state of neural stem cells [14]. Covello et al [15] confirmed that OCT-4, a stem cell transcription factor, is a target of
HIF-2a. HIF-2a can activate OCT- 4 to regulate the self-renew and differentiation of stem cells. Kaidi et al [16] also showed that Wnt/ $\beta$-catenin signaling pathway could interact with HIF-1a. Thus, there is definite evidence showing that hypoxia is related to the signaling pathways involving in the regulation of stemness of normal stem cells. Moreover, findings also demonstrate that the molecules in these signaling pathways also involve in the self-renew and differentiation of CSCs $[17,18]$. Axelson et al [19] found hypoxia could alter the expression of differentiation related genes in neuroblastoma cells and breast cancer cells, which promotes the de-differentiation of differentiated cancer cells and the acquisition of stem cell like features in these cells. Tavaluc et al [20] confirmed that hypoxia could increase the side population cancer cells. However, few studies have been conducted to investigate the influence of hypoxia on glioma cells and CSCs in glioma.

In our previous studies, results showed CAIX expression was related to the markers, invasion of stem cells and the prognosis of cancers. On the basis of these findings, we proposed that hypoxia could elevate the stemness of CSCs in glioblastoma, and our study comprehensively demonstrated that hypoxia was a niche of CSCs. Firstly, the influence of hypoxia on the growth of glioma cells was detected. Results showed hypoxia maintained the undifferentiated state, and normoxia promoted the differentiation of these cells. The growth curve was delineated, and cell cycle was detected. Results showed that hypoxia slowed down the growth and increased the percentage of the cells in the quiescent stage. Then, the biological behaviors of glioma cells were detected followed hypoxic treatment. The influence of hypoxia on the proliferation and migration of glioma cells was 
explored. Results indicated that hypoxia could promote the self-renewal and migration of these cells. Finally, western blot assay, PCR and immunofluorescence staining were employed to investigate the influence of hypoxia on the markers of CSCs in glioma and their differentiation. Findings indicated that hypoxia increased the expression of CD133 and nestin, markers of CSCs, but reduced the proportion of cells positive for GFAP, a marker for differentiation of stem cells. On the basis of influence of hypoxia on the function of glioblastoma cells, self-renew and differentiation of CSCs and expression of markers of stem cells, we concluded that hypoxia could de-differentiate the differentiated glioma cells and promote the acquisition of stemness in these cells. However, whether the elevation of stemness is attributed to the increase in CSCs in glioma due to de-differentiation of differentiated glioma cells or to the increase in proliferation of CSCs following hypoxia is still unclear, and more studies are required to elucidate it.

Taken together, our findings comprehensively demonstrate that hypoxia elevates the stemness of glioma cells in vitro, but in vivo studies are required and the specific mechanism underlying this effect is still poorly understood. In our future studies, in vivo studies and those on mechanism of this effect will be conducted aiming to identify the target for the treatment of glioma. Currently, the studies on CSCs are still in its infancy stage, and more studies are needed to elucidate the interaction between CSCs and their microenvironment.

\section{Acknowledgment}

The study was supported by National Natural Science Foundation of China (30872649).

\section{Competing Interests}

The authors have declared that no competing interest exists.

\section{References}

1. Penas-Prado M, Armstrong TS, Gilbert MR. Glioblastoma. Handbook of clinical neurology / edited by P.J. Vinken and G.W. Bruyn. 2012; 105: 485-506.

2. Thirant C, Galan-Moya EM, Dubois LG, et al. Differential proteomic analysis of human glioblastoma and neural stem cells reveals HDGF as a novel angiogenic secreted factor. Stem Cell. 2012; 30: 845-53.

3. Rossi DJ, Jamieson CH, Weissman IL. Stems cells and the pathways to aging and cancer. Cell 2008; 132: 681-96.

4. Keith B, Simon MC. Hypoxia-inducible factors, stem cells, and cancer. Cell 2007; 129: 465-72.

5. Yi L, Zhou ZH, Ping YF, et al. Isolation and characterization of stem cell-like precursor cells from primary human anaplastic oligoastrocytoma. Modern Pathol 2007;20: 1061-8.

6. Seo SK, Jeong HY, Park SG, et al. Blockade of endogenous B7-H1 suppresses antibacterial protection after primary Listeria monocytogenes infection. Immunology 2008; 123: 90-9.
7. Bruick RK. Oxygen sensing in the hypoxic response pathway: regulation of the hypoxia-inducible transcription factor. Genes Dev. 2003; 17: 2614-23.

8. Semenza GL. Hypoxia-inducible factor 1: oxygen homeostasis and disease pathophysiology. Trends Mol Med. 2001;7: 345-50.

9. Raval RR, Lau KW, Tran MG, et al. Contrasting properties of hypoxia-inducible factor 1 (HIF-1) and HIF-2 in von Hippel-Lindau-associated renal cell carcinoma. Mol Cell Biol. 2005; 25: 5675-86.

10. Ryan HE, Lo J, Johnson RS. HIF-1 alpha is required for solid tumor formation and embryonic vascularization. EMBO J. 1998; 17: 3005-15.

11. Carroll VA, Ashcroft M. Role of hypoxia-inducible factor (HIF)-1alpha versus HIF-2alpha in the regulation of HIF target genes in response to hypoxia, insulin-like growth factor-I, or loss of von Hippel-Lindau function: implications for targeting the HIF pathway. Cancer Res. 2006; 66: 6264-70.

12. Morrison SJ, Csete M, Groves AK, Melega W, Wold B, Anderson DJ. Culture in reduced levels of oxygen promotes clonogenic sympathoadrenal differentiation by isolated neural crest stem cells. J Neurosci 2000; 20: 7370-6.

13. Studer L, Csete M, Lee SH, et al. Enhanced proliferation, survival, and dopaminergic differentiation of CNS precursors in lowered oxygen. J Neurosci 2000; 20: 7377-83.

14. Gustafsson MV, Zheng $X$, Pereira $T$, et al. Hypoxia requires notch signaling to maintain the undifferentiated cell state. Dev Cell. 2005; 9: 617-28.

15. Covello KL, Kehler J, Yu H, et al. HIF-2alpha regulates Oct-4: effects of hypoxia on stem cell function, embryonic development, and tumor growth. Genes Dev. 2006; 20: 557-70.

16. Kaidi A, Williams AC, Paraskeva C. Interaction between beta-catenin and HIF-1 promotes cellular adaptation to hypoxia. Nat Cell Biol 2007; 9: 210-U113.

17. Rossi DJ, Jamieson CHM, Weissman IL. Stems cells and the pathways to aging and cancer. Cell 2008;132: 681-96

18. Keith B, Simon MC. Hypoxia-inducible factors, stem cells, and cancer. Cell 2007; 129: 465-72.

19. Axelson H, Fredlund E, Ovenberger M, Landberg G, Pahlman S. Hypoxia-induced dedifferentiation of tumor cells - A mechanism behind heterogeneity and aggressiveness of solid tumors. Semin Cell Dev Biol 2005; 16: 554-63.

20. Tavaluc RT, Hart LS, Dicker DT, El-Deiry WS. Effects of low confluency, serum starvation and hypoxia on the side population of cancer cell lines. Cell Cycle 2007; 6: 2554-62. 